\title{
Impacts of Human Body Shadowing in Wireless Body-Centric Communications
}

\author{
Mojaiana Synthia \\ Electronics and Communication Engineering Discipline \\ Khulna University
}

\begin{abstract}
In wireless body-centric communications, either the transmitter or the receiver, or both are located in close proximity to the human body. Thus, the Line-of-Sight (LOS) components of the radio signal are particularly susceptible to shadowing caused by human body. In earlier, the effect of human body shadowing was studied using $\kappa-\mu$ fading channel, where the resultant dominant signal components due to body shadowing effect was characterized by log-normal distribution and Nakagami-m distribution in different experiments. In this context, this article provides a comprehensive comparison of two aforementioned methods of human body shadowing effects in wireless body-centric communications. Here, the dominant component characterized by log-normal distribution is mentioned as shadowed log-normal model, whereas the Nakagami-m distribution dominant component model is mentioned as shadowed Nakagami-m model. Different wireless body-centric communications scenarios, namely on-body, off-body, and body-tobody, are tested separately with these two distribution methods. MATLAB based simulations indicate that the shadowed Nakagami-m distribution provides better fit to the empirical results in comparison of shadowed log-normal distribution. Moreover, the probability density function of shadowed Nakagami-m model provides numerical advantages over shadowed log-normal model.
\end{abstract}

Keywords: Log-normal distribution, Nakagami-m distribution, $\kappa-\mu$ fading, wireless body-centric communications

\section{Introduction}

In wireless radio communications, the LOS signal propagation is characterized by two physical mechanisms, namely multipath fading and shadowing. In multipath fading, the multipath components cause rapid and deep fading in displacements of few wavelengths or short time duration. This is also called fast fading or small-scale propagation [4]. The statistical model of multipath fading has mainly been presented by the Rice, Rayleigh, Nakagami-m, and Weibull distributions. However, the Nakagami-m distribution provides better fit to the field trials, particularly better fitting being found around the mean or median [1], [4]. While, in case of shadowing, the received signal fluctuates slowly around a mean in displacements of hundreds of wavelengths. Shadowing is encountered due to the temporal blockage of the LOS components between the transmitter and receiver. However, shadowing is commonly modeled statistically by the log-normal distribution [5].

On the other hand, the $\kappa-\mu$ fading distribution provides a general multipath model for LOS propagation scenarios, which can be used to represent the small-scale variation of the fading signal. This general distribution of $\kappa-\mu$ fading is controlled by two shape parameters, $\kappa$ and $\mu$, and represents some classical small-scale fading distributions, namely one-sided Gaussian, Rayleigh, Nakagami-m, and Rice distributions. In comparison with classical distributions, $\kappa-\mu$ distribution provides better fit to the experimental data. In $\kappa-\mu$ distribution, the statistics of the received signal are considered 
in clusters of waves. Within each clusters, a dominant signal component with arbitrary power may exist, and the dominant signal is assumed to be deterministic. Also, in $\kappa-\mu$ fading model, the propagation environment is considered as homogeneous.

However, in wireless body-centric communications, the transmitter or receiver, or both of the nodes are located at human body, thus the LOS components are shadowed by body matter situated in the path of propagation. Also, the physiological and biomechanical processes of non-homogeneous human body indicate that the resultant shadowing of the LOS signal produce randomly variable dominant components. The article in [2] studied the human body shadowing effect on wireless body-centric communications, where the resultant dominant components due to body shadowing was assumed to follow lognormal distribution. In [2], it was also shown than the statistical model is good fit to the experimental data. While, in [3], the Nakagami-m distribution was considered to characterize the dominant components due to the human body shadowing effects on device-to-device communications, and the model also indicated a good fit to the field measurements. In this project, both of the log-normal and Nakagami-m distributions are applied to represent the distribution of the resultant dominant components in effect of human body shadowing in wireless body-centric communications.

The rest of the article is organized as follows. Section 2 presents the shadowing scenarios in on-body, off-body, and body-to-body communications channels. The statistical channel distribution for shadowed log-normal and shadowed Nakagami-m model are presented in Section 3. The comparison of two models with field trials of [2] is shown in Section 4 in terms of MATLAB based simulations. Finally, Section 5 draws conclusion of this article by summarizing the other pros and cons of the subjected models.

\section{Shadowing Scenarios in Body-Centric Communications}

Let us consider a wireless Non Line-of-Sight (NLOS) body-centric communications link, thus no direct LOS path exist between the transmitter and the receiver. Then, the communication likely to be maintained by a number of dominant components such as diffracted, scattered, reflected, and trapped surface waves due to human body shadowing, as well as multipath components due to environmental effects [6]. However, the complicated geometric and dielectric properties of the nonhomogeneous human body [6] indicates that the resultant dominant components are also subjected to be random shadowing. Figure 1 illustrates the shadowing scenarios for three main types of bodycentric communications channels [2].

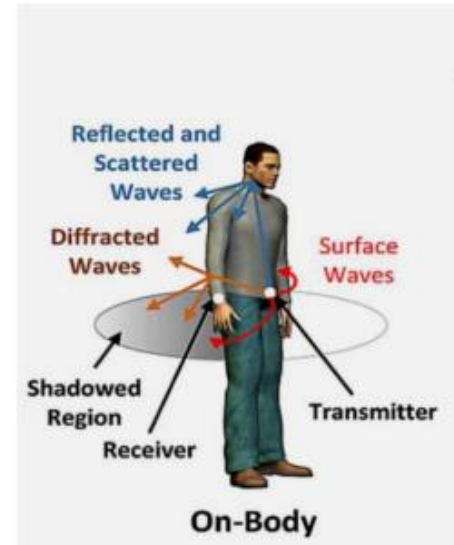

(a)

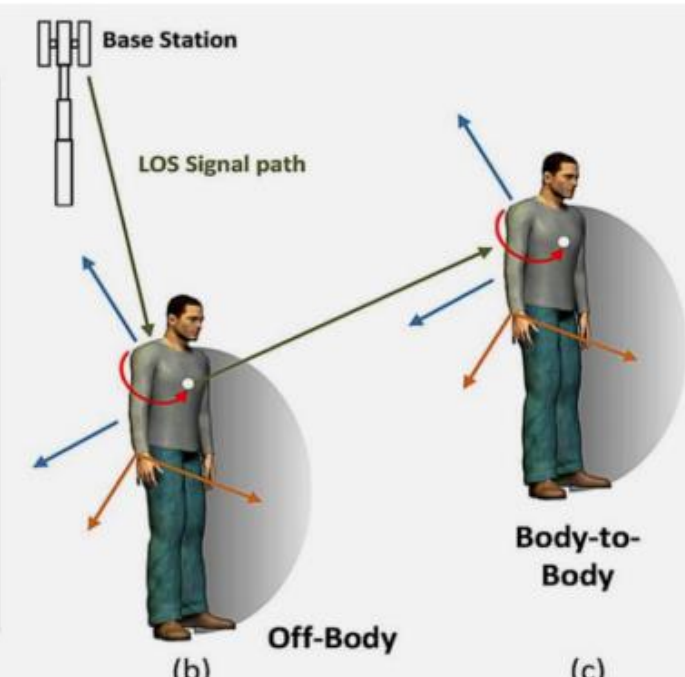

(b) (c)

Figure 1. Illustrations of Various Body-centric Communications Channels 
In case of on-body communications, both of the transmitter and receiver are located at same body. Thus, the wireless channel between the transmitter and receiver is highly susceptible to shadowing caused by human tissue and moving limbs. As shown in Figure 1(a), if the subject randomly walks, then the wireless channels between the front-leftwaist to right-wrist, and front-left-waist to right-leg will be quasi-periodic shadowing due to the movement of wrist and leg.

In off-body communications, one transceiver is located at human body, and communicates with a non-body-worn base station located at separate place. Therefore, the wireless channel of off-body communications is also susceptible to frequent shadowing due to the movement of subjected user, and the effects of environments as well. On the other hand, in case of body-to-body communications, the effect of shadowing is apparently double than the off-body communications, since the transmitter is located at one body while receiver is at another body. Figure 1(b) and Figure 1(c) demonstrates the body shadowing effect of off-body and body-to-body communications, respectively.

\section{Statistical Model of Shadowing in Body-centric Communications}

The physical propagation model of body-centric communications discussed in Section II represents the same propagation model as $\kappa-\mu$ fading model [1], except that the dominant components are also varied randomly instead of deterministic. According to the $\kappa-\mu$ distribution, the complex received signal envelope, $R \exp (j \theta)$, can be written as the sum of the resultant scattered waves $(W)$ and the resultant dominant components $(\Delta)$ such that

$$
R \exp (j \theta)=W \exp (j \phi)+\Delta \exp \left(j \phi_{0}\right)
$$

Where, $\phi$ is the stationary random phase process associated with scattered waves, and $\phi_{0}$ is the phase of the resultant dominant components distributed over the range $(-\pi, \pi)$. Also, the resultant scattered wave (W) follows Nakagami-m distribution as,

$$
f_{W}(w)=\frac{2 \mu^{\mu}}{\Omega^{\mu} \Gamma(\mu)} \exp \left(-\frac{\mu w^{2}}{\Omega}\right) w^{(2 \mu-1)}
$$

In addition, if the dominant components $(\Delta)$ are assume to be deterministic, then the complex received signal $(\mathrm{R})$ follows the $\kappa-\mu$ distribution $[1]$ as,

$$
f_{R \mid \Delta}(r \mid \delta)=\frac{r^{\mu}}{\sigma^{2} \delta^{\mu-1}} \exp \left(-\frac{r^{2}+\delta^{2}}{2 \sigma^{2}}\right) I_{\mu-1}\left(\frac{\delta r}{\sigma^{2}}\right)
$$

Where, $\delta, \sigma$ and $\mu$ are parameterized in terms of $\kappa-\mu$ distribution. The $I_{\mu-1}(\bullet)$ is the modified Bessel function of the first kind, order $\mu-1$. Also, $\kappa$ is the power ratio of the dominant components and the scattered waves as,

$$
\kappa=\frac{\delta^{2}}{2 \mu \sigma^{2}}
$$

The mean power of (4) can be calculated as,

$$
E\left[R^{2}\right]=\hat{\mathrm{r}}^{2}=\delta^{2}+2 \mu \sigma^{2}
$$

\subsection{Shadowed Log-normal Model}

Let us consider that the dominant components are follow log-normal distribution such that

$$
f_{\Delta}(\delta)=\frac{1}{\delta \beta \sqrt{2 \pi}} \exp \left(-\frac{(\ln \delta-\alpha)^{2}}{2 \beta^{2}}\right)
$$

Where, $\alpha$ and $\beta$ are the location and scale parameters, respectively, of the log-normal distribution. Therefore, with log-normal distribution of dominant components, the received complex signal of (3) can be written as [2],

$$
f_{R}(r)=\frac{r^{\mu}}{\beta \sigma^{2} \sqrt{2 \pi}} \int_{0}^{\infty} \frac{1}{\delta^{\mu}} \exp \left(-\frac{\sigma^{2}(\ln \delta-\alpha)^{2}+\beta^{2}\left(r^{2}+\delta^{2}\right)}{2 \beta^{2} \sigma^{2}}\right) I_{\mu-1}\left(\frac{\delta r}{\sigma^{2}}\right) d \delta
$$

Equation (7) is the PDF of shadowed log-normal body-centric communications channels. 


\subsection{Shadowed Nakagami-m Model}

Under this model, the dominant components of the received complex signal are assumed to follow Nakagami-m distribution as,

$$
f_{\Delta}(\delta)=\frac{2 m^{m}}{\Omega^{m} \Gamma(m)} \delta^{(2 m-1)} \exp \left(\frac{-m \delta^{2}}{\Omega}\right)
$$

Where, $\Gamma(\bullet)$ is the gamma function, and $m=\frac{E\left[\Delta^{2}\right]}{\operatorname{var}\left[\Delta^{2}\right]}$ is the Nakagami parameter with variance of $\operatorname{var}\left[\Delta^{2}\right][1]$. In this instance, $\Omega=E\left[\Delta^{2}\right]$ is the average power of the resultant dominant components. The parameter $m$ is allowed to take any value in the range $m \geq 0$, where $m \rightarrow 0$ corresponds to complete shadowing of the resultant dominant component and $m \rightarrow \infty$ corresponds to no shadowing of the resultant dominant component. Therefore, the distribution of the received complex signal under shadowed Nakagami-m model can be written as [3]

$$
f_{R}(r)=\frac{r^{2 \mu-1}}{2^{\mu-1} \sigma^{2} \Gamma(\mu)}\left(\frac{2 m \sigma^{2}}{\Omega+2 m \sigma^{2}}\right)^{m} \exp \left(-\frac{r^{2}}{2 \sigma^{2}}\right) \times{ }_{1} F_{1}\left(m ; \mu ; \frac{r^{2} \Omega}{2 \sigma^{2}\left(\Omega+2 m \sigma^{2}\right)}\right)
$$

Now, from equation (4) and equation (5), $\sigma^{2}$ can be expressed in terms $\hat{\mathrm{r}}, \mu$, and $\kappa$ of as,

$$
\sigma^{2}=\frac{\hat{\mathrm{r}}^{2}}{2 \mu(1+\kappa)}
$$

Putting the value of $\sigma^{2}$ in equation (9), the PDF of shadowed Nakagami-m model in body-centric communications can write as,

$$
\begin{aligned}
& f_{R}(r)= \frac{2 r^{2 \mu-1}}{\Gamma(\mu)}\left(\frac{\mu(1+\kappa)}{\hat{\mathrm{r}}^{2}}\right)^{\mu}\left(\frac{m \hat{\mathrm{r}}^{2}}{\mu(1+\kappa) \Omega+m \hat{\mathrm{r}}^{2}}\right)^{m} \times \\
& \quad \exp \left(-\frac{\mu(1+\kappa) r^{2}}{\hat{\mathrm{r}}^{2}}\right) \times{ }_{1} F_{1}\left(m ; \mu ; \frac{(\mu(1+\kappa) r)^{2} \Omega}{\hat{\mathrm{r}}^{2}\left(\mu(1+\kappa) \Omega+m \hat{\mathrm{r}}^{2}\right)}\right)
\end{aligned}
$$

Where, $\times_{1} F_{1}(\bullet ; \bullet ; \bullet)$ is the confluent hypergeometric function.

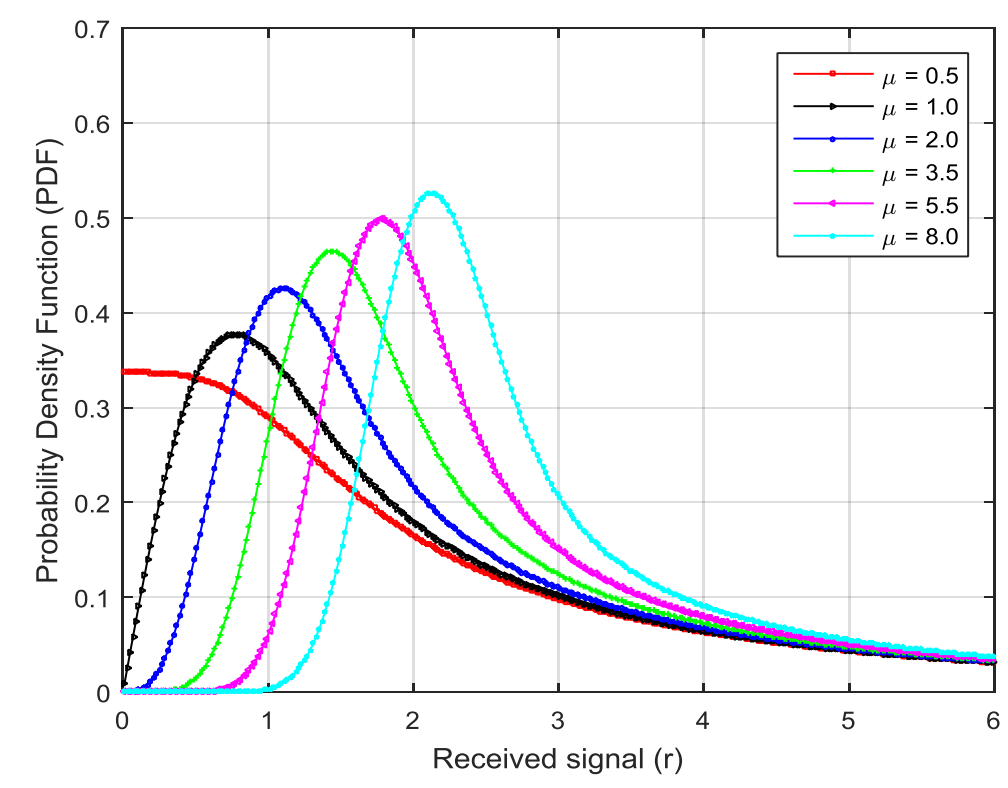

Figure 2. PDFs of shadowed Log-normal Model for Increasing Value of $\mu$. The Value $\alpha, \beta$, and $\delta$ are given in [2]

The probability density function of the shadowed log-normal model and shadowed Nakagami- model is shown in Figure 2 and Figure 3, respectively. 


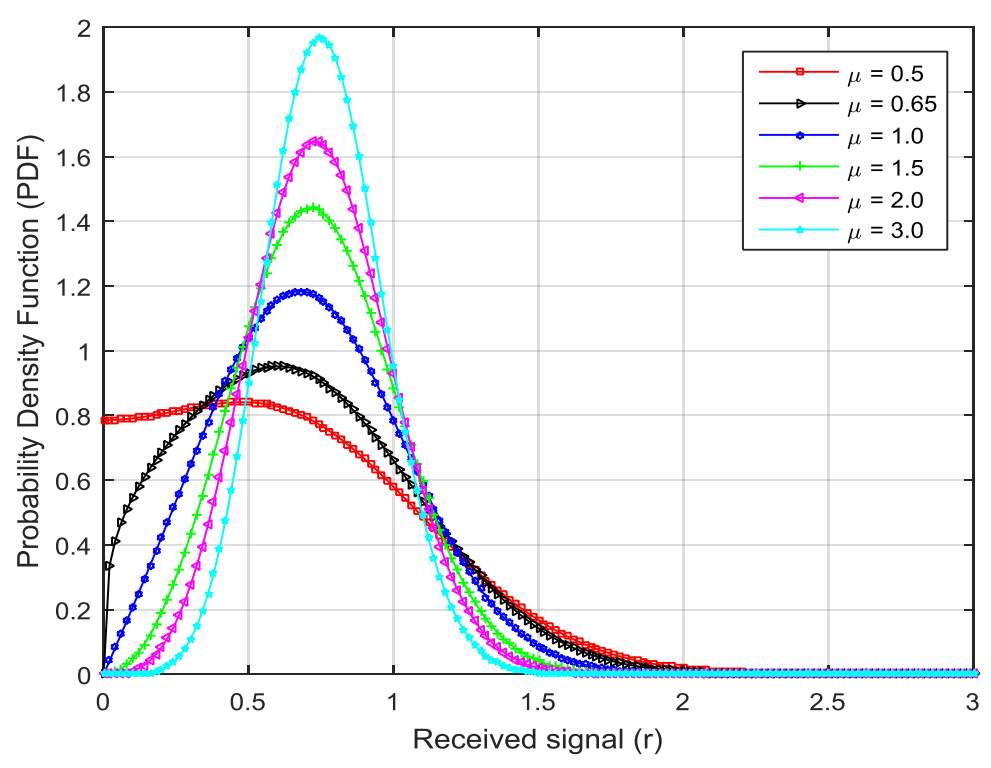

Figure 3. PDFs of Shadowed Nakagami-m Model for Increasing Value of $\mu$. The Value of $\boldsymbol{\kappa}$ is found in [3], and value of $m$, and $\Omega$ are given in [7], while $\hat{\mathrm{r}}=\mathbf{1}$

\section{Channel Characteristics with two Shadowed Models}

In the section, the body-centric communications channels presented in [2] are analyzed using shadowed log-normal model presented in (7) and shadowed Nakagami-m model presented in equation (11). Then, two models are closely compared with the empirical results presented in [2]. All the parameters are estimated using maximum likelihood estimation (mle) method used in [2] and [3].

\subsection{On-Body Channels}

Figure 4 shows the PDFs of experimental data of on-body channels in an anechoic chamber, and the statistical PDFs of shadowed log-normal model tested in [2].

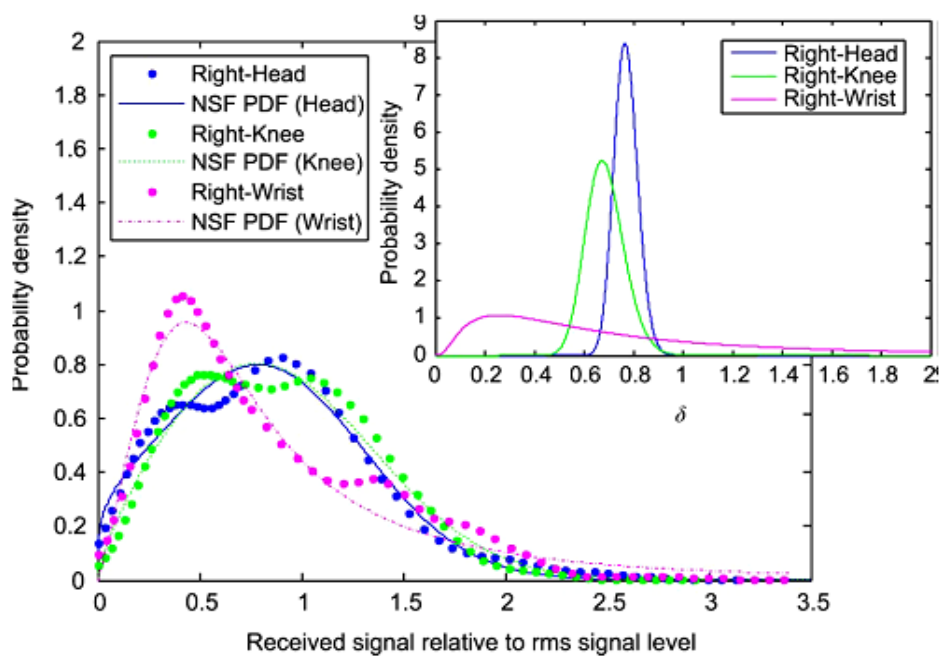

Figure 4. PDFs of Empirical Data and Shadowed Log-Normal Model for onBody Channels 
While, Figure 5 shows the statistical PDFs of both of the shadowed Nakagami-m and shadowed log-normal model. The dimension of the transmitter and receiver are shown in [2]. If we compare the statistical PDFs of Figure 5 with the experimental results of Figure 4 , it is clear that the shadowed Nakagami-m model provides better fit to the empirical data than the shadowed log-normal model, particularly around the mean or median. All three on-body links, namely front-left-waist to right-head, front-left-waist to right-knee, and front-left-waist to right-wrist, equally supports the above arguments.

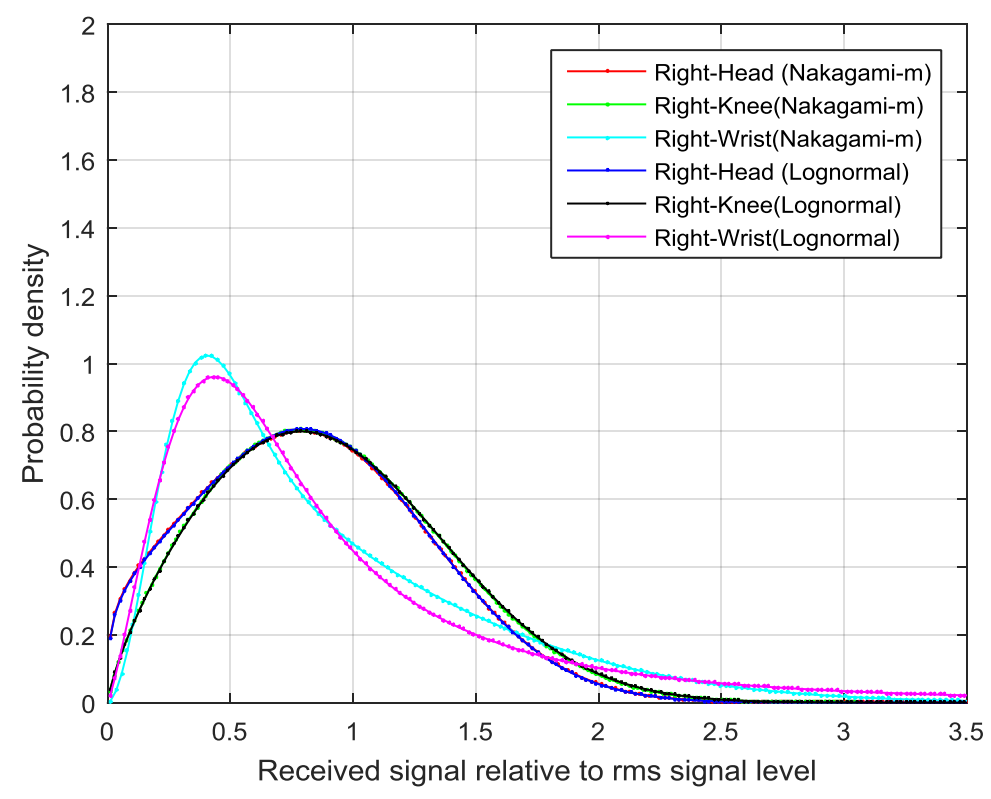

Figure 5. PDFs of Shadowed Nakagami-m and Shadowed Log-Normal Model for on-Body Channels

\subsection{Off-Boy Channel}

In off-body channels, the PDFs of shadowed log-normal distribution along with the filed data are shown in Figure 6 [2].

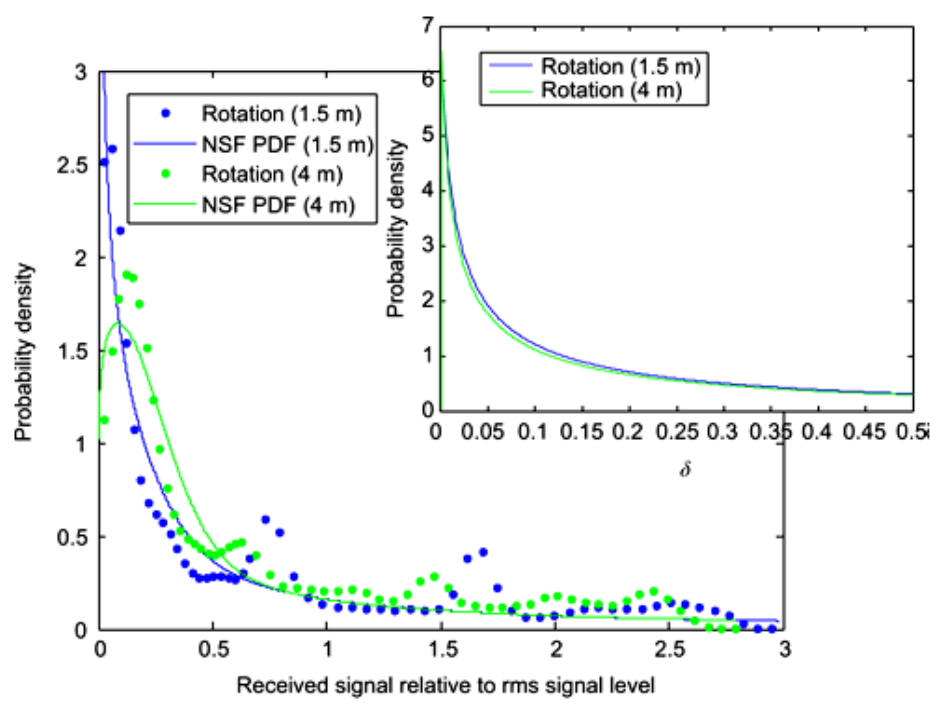

Figure 6. PDFs of Empirical Data and Shadowed Log-Normal Model for OffBody Channels 
In this case, the transmitter are located at human body, and performed $360^{\circ}$ counter clockwise rotation at a distance $1.5 \mathrm{~m}$ and $4 \mathrm{~m}$ away from a non-body-worn receiver. The experiment was also performed in anechoic chamber mentioned in [2], and the dimension of transceiver is also shown [2]. However, the statistical PDFs of the shadowed Nakagami-m and shadowed log-normal model, derived by using the parameters from field data of Figure 6, are presented in Figure 7. By observing Figure 6 and Figure 7, it is clearing found that the shadowed Nakagami-m model is better fitted to the field results compare to shadowed log-normal model for off-body communications channels.

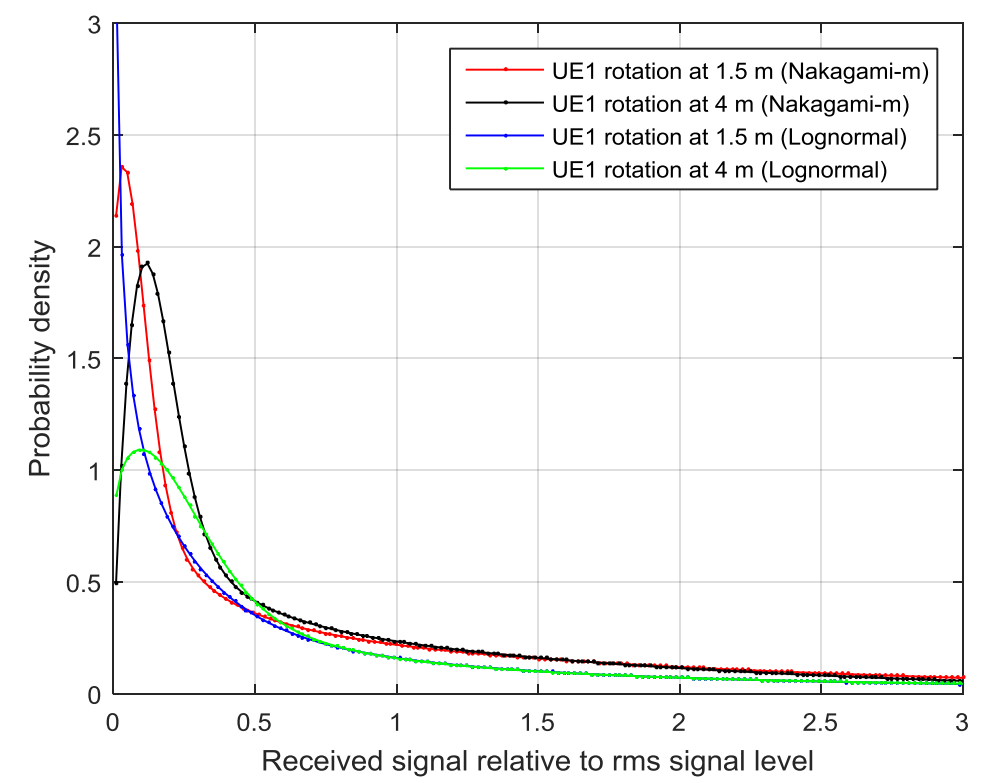

Figure 7. PDFs of Shadowed Nakagami-m and Shadowed Log-Normal Model for Off-Body Channels

\subsection{Body-to-Body Channel}

Unlike the on-body and off-body channels, the field trial of body-to-body channels was performed in outdoor environment mentioned in [2], and the dimension of transceiver is also shown in [2].

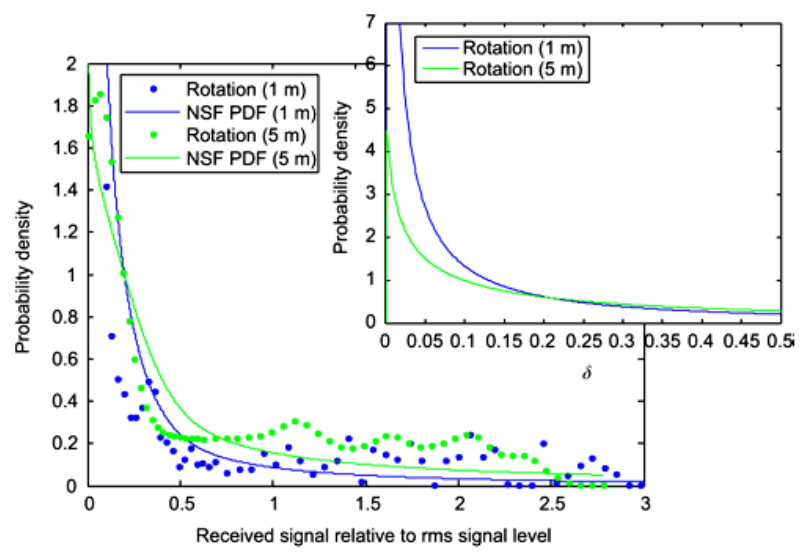

Figure 8. PDFs of Empirical Data and Shadowed Log-Normal Model for Body-To-Body Channels 
In body-to-body channels, a body-worn user equipment (UE1) performed $360^{\circ}$ counter clockwise rotation at a distance $1 \mathrm{~m}$ and $5 \mathrm{~m}$ away from another body-worn user (UE2). The PDFs of field data and estimated PDFs using shadowed log-normal distribution are shown in Figure 8 [2]. However, the statistical PDFs of the shadowed Nakagami-m and shadowed log-normal model, derived by using parameters from field data of Figure 8, are revealed in Figure 9. In similar to on-body and off-body channels, Figure 8 and Figure 9 indicates that the shadowed Nakagami-m model is better fitted to the field trials in comparison of shadowed log-normal model for body-to-body communications channels.

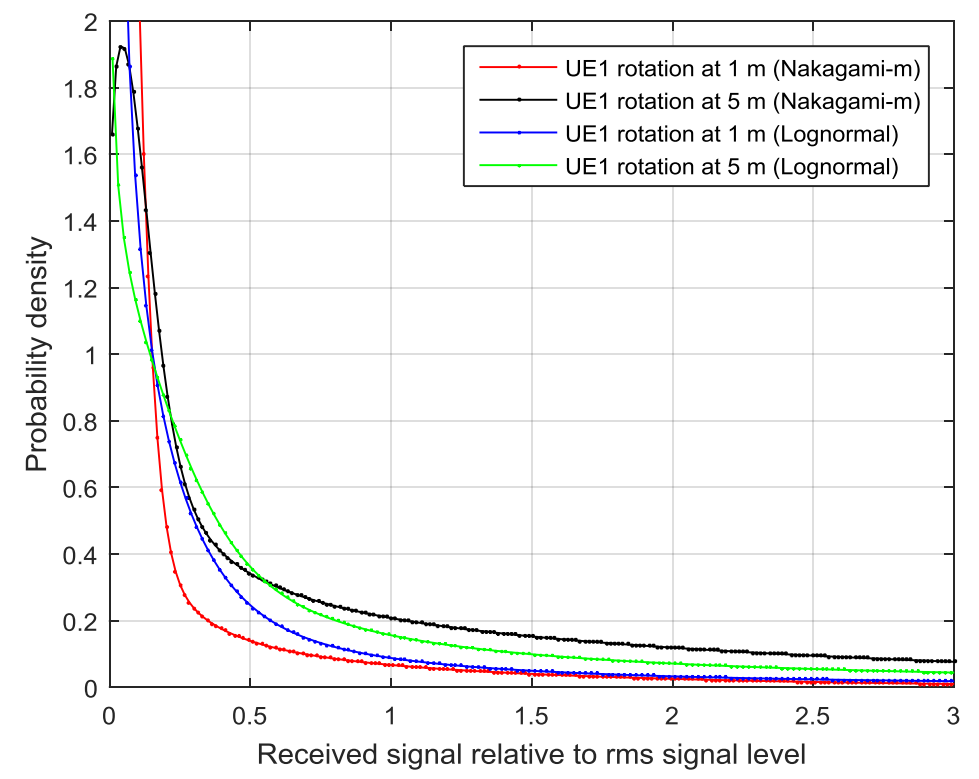

\section{Figure 9. PDFs of Shadowed Nakagami-M and Shadowed Log-Normal Model for Body-To-Body Channels}

\section{Conclusion}

In this article, the comparison of shadowed log-normal model of [2] and shadowed Nakagami-m model of [3] has been investigated in the area of wireless body-centric communications. Through MATLAB-based simulations, it has been proved that the shadowed Nakagami-m model is better fitted to the field trails in comparison of shadowed log-normal model.

In addition, the statistical distribution of shadowed Nakagami-m model provides closed-form expression. Along with the closed-form probability density function, the moments, and moment-generating function of shadowed Nakagami-m model are also easily derivable [3]. While, no closed-form expression exists for probability density function of shadowed log-normal model, and the integral must be evaluated numerically.

\section{References}

[1] M. D. Yacoub, "The $\kappa-\mu$ Distribution: A General Fading Distribution", IEEE Antenna Propagation Magazine, vol. 49, no. 1, (2007), pp. 1427-1431.

[2] S. L. Cotton, "A Statistical Model for Shadowed Body-centric Communications Channels: Theory and Validation”, IEEE Transactions on Antennas and Propagation, vol. 62, no. 3, (2014), pp. 1416-1424.

[3] S. L. Cotton, "Human Body Shadowing in Cellular Device-to-device Communications: Channel Modeling using the Shadowed $\kappa-\mu$ Fading Model", under revision, IEEE Journal on Selected Areas in Communications, vol. 33, no. 1, (2015).

[4] L. Rubio, J. Reig and N. Cardona, "Evaluation of Nakagami Fading Behavior Based on Measurements in Urban Scenarios", International Journal of Electronics and Communications, vol. 61, no. 2, (2007), pp. 135-138. 
[5] Y. Wang, I. B. Bonev, J. O. Nielsen, I. Z. Kovacs and G. F. Pedersen, "Characterization of the Indoor Multi-Antenna Body-to-Body Radio Channel”, IEEE Trans. On Antennas and Propagation, vol. 57, no. 4, (2009), pp. 972-979.

[6] G. A. Conway and W. G. Scanlon, "Antennas for Over-Body-Surface Communication at 2.45 GHz", IEEE Trans. Antennas Propagation, Special Issue on Antennas and Propagation on Body-centric Wireless Communications, vol. 57, (2009), pp. 844-855.

[7] L. W. C. Abdi, M. S. Alouini and M. Kaveh, "A New Simple Model for Land Mobile Satellite Channels: First- and Second-Order Statistics", IEEE Trans. Wireless Communication, vol. 2, no. 3, (2003), pp. 519-528.

[8] M. D. Yacoub, “The $\kappa-\mu$ and the $\eta-\mu$ Distribution”, IEEE Antenna Propagation Magazine, vol. 49, no. 1, (2007), pp. 68-81. 
International Journal of Future Generation Communication and Networking Vol. 9, No. 6 (2016) 